\title{
Nanoindentation of a circular sheet of bilayer graphene
}

\author{
M. Neek-Amal ${ }^{1}$ and F. M. Peeters ${ }^{2}$ \\ ${ }^{1}$ Department of Physics, Shahid Rajaei University, Lavizan, Tehran 16785-136, Iran \\ ${ }^{2}$ Departement Fysica, Universiteit Antwerpen, Groenenborgerlaan 171, B-2020 Antwerpen, Belgium \\ (Received 12 October 2009; revised manuscript received 19 April 2010; published 11 June 2010)
}

\begin{abstract}
Nanoindentation of bilayer graphene is studied using molecular-dynamics simulations. We compared our simulation results with those from elasticity theory as based on the nonlinear Föppl-Hencky equations with rigid boundary condition. The force-deflection values of bilayer graphene are compared to those of monolayer graphene. Young's modulus of bilayer graphene is estimated to be $0.8 \mathrm{TPa}$ which is close to the value for graphite. Moreover, an almost flat bilayer membrane at low temperature under central load has a $14 \%$ smaller Young's modulus as compared to the one at room temperature.
\end{abstract}

DOI: 10.1103/PhysRevB.81.235421

PACS number(s): $62.25 .-\mathrm{g}$

\section{INTRODUCTION}

Graphene is an almost flat one-atom-thick layer of carbon atoms that are densely packed in a honeycomb crystal lattice. Most of previous studies concerned the electronic properties of graphene but these two-dimensional graphene membranes have also exceptional mechanical properties. ${ }^{1-3}$ What makes graphene so exceptional is that it stays strong and stiff even up to a single atomic layer which differs from most materials whose mechanical properties substantially deteriorate as they are made thinner. These mechanical properties make graphene suitable for applications in, e.g., pressure sensing. A nanomembrane made of graphene, the ultimate downscaling limit of a nanoelectromechanical system, acts as a sensor that is predicted to detect ultrasmall masses and being at the same time extremely robust against long-term wear.

Recently, a nonlinear behavior of stress-strain response of monolayer graphene was studied by Lee et al. ${ }^{4}$ using atomic force microscopy (AFM). They measured Young's modulus and found that graphene is a strong material like diamond. Furthermore, linear force-displacement curves were measured in Refs. 5-7. They obtained an effective spring constant for a micron-size monolayer graphene sheet equal to $0.2 \mathrm{~N} / \mathrm{m}$ (Ref. 7) and for a suspended micron-size sheet of multilayer graphene with thicknesses in the range $2-8 \mathrm{~nm}$ and found values in the range $1.0-5.0 \mathrm{~N} / \mathrm{m} .{ }^{5}$ Moreover, monolayer graphene has a negative thermal expansion up to $900 \mathrm{~K}$ and its Young's modulus increases with temperature up to $900 \mathrm{~K} .{ }^{8}$ These anomalous properties are a consequence of the strong anharmonicity in graphene. Most of these properties can be explained by traditional elasticity theory and statistical physics. 5

The aim of the present paper is to investigate if two van der Waals coupled graphene layers, also called bilayer graphene, has similar improved mechanical properties as monolayer graphene.

There are three common theoretical methods to study the indentation of a graphene sheet, finite-element method, continuum mechanics, and molecular mechanics simulations. ${ }^{9-12}$ These methods lead to different results for the forcedeflection curve and for the graphene deformation profiles. ${ }^{11}$ The discrepancy originates from the predominant bond- stretching mode predicted by the molecular mechanics model and a bending to stretching transition process under increasing deflection predicted by continuum mechanics. Nowadays various properties are measured on micron-size circular graphene ${ }^{4}$ while computational studies on nanoindentation of graphene typically are limited to nanosize systems. In a typical experimental setup, the graphene sheet is indented by a tip from an AFM.

An important issue in simulating nanoindentation of graphene is the type of interatomic potentials between the carbon atoms in the sheet and between the carbon atoms and the atoms of the tip. In recent studies on nanoindentation of graphene, several models were used. Xu et al. ${ }^{11}$ used 1392 atoms (a circular graphene sheet with radius $3.25 \mathrm{~nm}$ ) in their atomic simulation and obtained the elasticity response of such a nanocircular sheet of graphene under central load. The nanoindentation was realized by moving down the center of the sheet by hand. On the other hand, Hemmasizadeh et al. ${ }^{10}$ compared the results for the force-deflection curve obtained from a continuum elasticity $\operatorname{model}^{13}$ and finiteelement method calculations for two circular and hexagonal continuum plates of size $11 \mathrm{~nm}$ and showed that in the large deflection regime, there are important differences between these two approaches. This difference roots in the effect of exerting a concentrated force so that the difference reduced to half when a spherical indenter with radius $10 \AA$ was used in the finite-element method calculation.

Medyanik et al. ${ }^{9}$ studied the nanoindentation of a hexagonal graphene sheet and multilayered graphite sheets by employing a quasistatic formulation of the method of multiscale boundary conditions. They showed that their method of multiscaling gives good results in comparison to full domain atomistic simulations based on molecular mechanics simulations. They used repulsive interactions to model the spherical indenter. The number of atoms in the reduced domain was 2646 for single circular graphene. Depending on the size of the reduced domain, their results for multilayer graphene showed an increased force-deflection dependence as compared to monolayer graphene.

In this paper, we study the mechanical properties of bilayer graphene for three different sizes and compare the mechanical response of bilayer graphene to the one of monolayer graphene. The Young's modulus of bilayer graphene is estimated by using the predictions from the theory of elas- 
ticity for a loaded plate in the large deflection regime. Furthermore, the temperature dependence of the nanoindentation of bilayer graphene is investigated.

This paper is organized as follows. In Sec. II, we will introduce the atomistic model, the simulation method and the results from elasticity theory. Section III contains the numerical results and in Sec. IV, we will present our conclusion.

\section{THEORY AND MODEL}

We have used classical atomistic molecular-dynamics simulation to simulate the nanoindentation of a suspended sheet of bilayer graphene. The number of carbon atoms, i.e., $N_{b}$, varies from 35688 to 152308 which are equivalent to surfaces with radius $R=12 \mathrm{~nm}$ and $R=25 \mathrm{~nm}$, respectively. A rigidly clamped boundary condition was imposed. In our simulations, the indenter consists of $N_{t}=371$ atoms in a fcc structure (lattice constant equal to $3.92 \AA$ ) and it is assumed rigid during our simulation. The shape of the indenter was chosen as a square-based pyramid. The area of the bottom surface (square) of the tip is $2.02 \mathrm{~nm}^{2}$ and the top one has an area of $10.24 \mathrm{~nm}^{2}$. Initially, the coordinates of all atoms in each layer are put in a flat surface of a honeycomb lattice with nearest-neighbor distance equal to $0.142 \mathrm{~nm}$ while the upper layer is shifted along armchair direction by $0.142 \mathrm{~nm}$ above the bottom layer. The separation between the two layers is $3.5 \AA$. The initial velocities in each direction were extracted from a Maxwell-Boltzman distribution for the given temperature. We simulated the system at room temperature 300 and $20 \mathrm{~K}$ by employing a Nos'e-Hoover thermostat. The Brenner's bond-order potential ${ }^{14,15}$ was used for the carbon-carbon interaction and a Lennard-Jones (LJ) potential $U(r)=4 \varepsilon\left\{(\sigma / r)^{12}-(\sigma / r)^{6}\right\}$ for the indenter-graphene interaction and the interaction between the two graphene layers. In the LJ potential, $\sigma$ is the distance at which the potential is zero and $\epsilon$ is the depth of the potential well. The van der Waals interaction between the two graphene layers was modeled by a LJ interaction with $\varepsilon_{\mathrm{C}}=2.84 \mathrm{meV}$ and $\sigma_{\mathrm{C}}$ $=3.4 \AA$. For the interaction between the tip and graphene, we used the LJ parameters for $\mathrm{Pt}$ atoms with $\varepsilon_{\mathrm{Pt}}$ $=68.3 \mathrm{meV}$ and $\sigma_{\mathrm{Pt}}=2.54 \AA .{ }^{16}$ For a two-component system, as studied here, the parameters for the mixed interaction between the two type of atoms can be estimated by the simple average $\sigma_{\mathrm{C}-\mathrm{Pt}}=\left(\sigma_{\mathrm{C}}+\sigma_{\mathrm{Pt}}\right) / 2$ and $\varepsilon_{\mathrm{C}-\mathrm{Pt}}=\sqrt{\varepsilon_{\mathrm{C}} \cdot \varepsilon_{\mathrm{Pt}}}$ suggested by Steel et al. ${ }^{17}$ To save computational time, we truncated the LJ potential at the cutoff distance of $r_{c}=3.5 \sigma$. Note that the LJ potential is a simple choice for modeling the interaction between two layers. ${ }^{18}$ To obtain more accurate results, one can use other potentials such as a Morse potential or other force fields. ${ }^{16}$ At the start of our simulation, the position of the lowest atoms of the tip are located a few angstrom, i.e., $\approx 3.4 \AA$ above the upper graphene layer. After equilibrating the system during 50000 time steps, the indenter is pushed down slowly with $\delta=0.2 \AA$ in a time span of $5000 \Delta t$ which is equivalent to a velocity of $8 \mathrm{~m} / \mathrm{s}$, where $\Delta t=0.5 \mathrm{fs}$ is the time step in our simulation. To avoid unphysical effects due to the time step, the indentation step, $\delta$, was chosen to be small with respect to the force cutoff
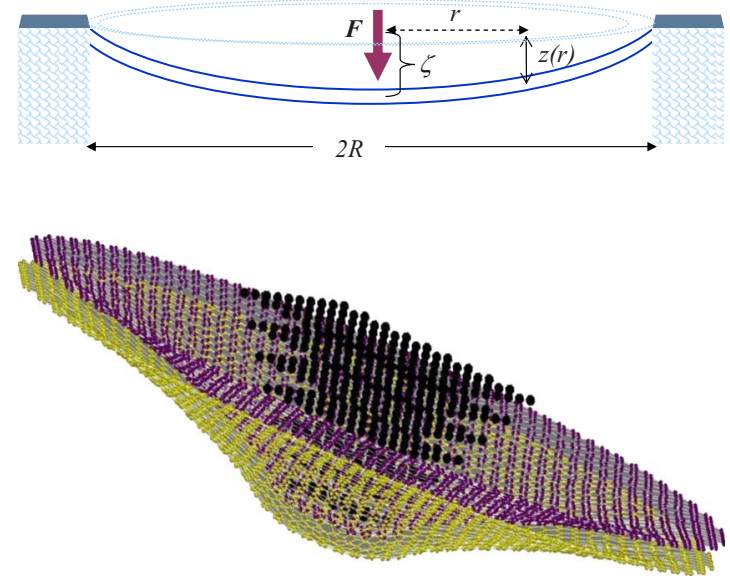

FIG. 1. (Color online) Top panel: schematic model of the suspended bilayer graphene with rigidly clamped boundary conditions. Bottom panel: a snapshot of an atomistic indenter over a circular bilayer graphene with $R=6 \mathrm{~nm}$ and clamped boundary conditions.

length, $r_{c}$, for the interatomic potential (e.g., to prevent a sudden change in the force, etc.).

The considered size of the system $(\sim n m)$ is larger than the deflection value $(\sim \AA)$, and in addition, the thickness of the sheet is smaller than the amount of deflection. Therefore, nonlinear elasticity theory ${ }^{19}$ for a circular flake in the large deflection limit along the $z$ direction is applicable. The energetics of such a circular flake in the limit of large deflection is considered including both bending and stretching energies. ${ }^{19}$ The condition of minimum energy for the flake yields the Föppl equation. The solution of the equation is obtained by using the Hencky transformation. The governing equations in planar-polar coordinates are as follows:

$$
\begin{gathered}
r \frac{d}{d r}\left[\frac{d}{r d r}\left(r^{2} \sigma_{r}\right)\right]=-\frac{t E}{2}\left(\frac{d z}{d r}\right)^{2}, \\
\sigma_{r} \frac{d z}{d r}=-\frac{F}{2 \pi r},
\end{gathered}
$$

where $r$ is the radial position, $R$ is the radius of the circular plate as shown in Fig. 1, $z(r)$ is the deflection at radial position $r, t$ is the thickness of the plate, $E$ is Young's modulus, $\sigma_{r}$ is the radial stress of the flake, and $F$ is the concentrated load on the flake. We are interested in the situation of a rigidly clamped boundary condition or fixed boundary condition in the absence of residual stress, i.e., $z=0$ at $r=0$. Expressions given by Eq. (1) are nonlinear equations, however they can be solved analytically in the special case of fixed boundary condition. ${ }^{20}$ In general, the solution is given by $^{20}$

$$
F=\frac{\pi E t}{4 R^{2}} \frac{1}{G(\nu)} z(r)^{3},
$$

where $G(\nu)$ is a complicated function of the Poisson ratio, $\nu$. $G(\nu)$ has almost a linear dependence on the Poisson's ratio of the desired system and varies from 0.9 for $\nu \simeq 0.0$ to 0.65 for $\nu \simeq 0.5$. 

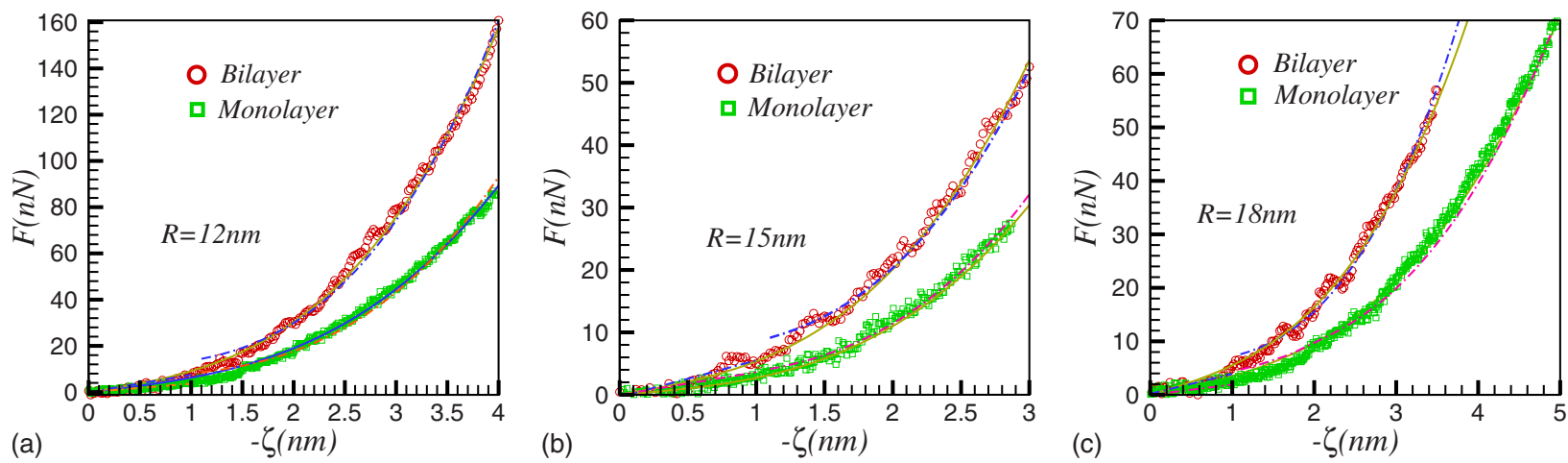

FIG. 2. (Color online) Load force as a function of displacement for three different radii. The analytical expression Eq. (3) is fitted and shown by the solid curves. The dashed-dotted curves refer to two separate fits for small and large deflections. For comparative purposes, we show also the results of monolayer graphene and corresponding fits (Ref. 23).

$\zeta$ is taken as the deflection of the bilayer graphene at $r$ $=0$, i.e., $\zeta=z(0)$. It is the difference between the center of mass of the central point of indented bilayer graphene and the first central nonindented equilibrium position of the bilayer (at $r=0)^{19}$ (see Fig. 1). During indentation, because the using of the LJ potential, the equilibrium distance between the lowest atoms of the tip and the central point of the bilayer is on the order of $\sigma_{\mathrm{C}-\mathrm{Pt}}$. Surprisingly, our computer simulations confirm this behavior which we will discuss in the next section. The force-displacement curves have been measured recently by Lee et al. ${ }^{4}$ and they showed that it can be approximated by a simple polynomial function having a linear and a cubic term,

$$
F=a \zeta+b \zeta^{3} .
$$

When the bending stiffness is negligible and the load is small the force deflection can be approximated by the linear term while the second term dominates for large deflection. This behavior was studied both theoretically and experimentally in Refs. 4, 13, and 21.

\section{NUMERICAL RESULTS}

Figure 1 gives a schematic view of the system showing the indenter and the clamped circular bilayer graphene and a snapshot of an atomistic indenter over a circular bilayer graphene with clamped boundary conditions. The $z$ component of the forces from the bilayer graphene atoms on the indenter are calculated by summing over the total reaction forces,

$$
F=\sum_{i=1}^{N_{t}} \sum_{j=1}^{N_{b}} F_{i j}^{z}=24 \varepsilon \sum_{k=1}^{2}\left\{\sum_{i=1}^{N_{t}} \sum_{j=1}^{N_{b}}(-1)^{k} 2^{k-1}\left(\frac{\sigma}{r_{i j}}\right)^{6 k} \frac{z_{i j}}{r_{i j}^{2}}\right\} .
$$

Often in molecular-dynamics simulations, one approximates the above sums by including only the nearest neighbors in order to reduce the number of interactions which is accurate in the case of short-range potentials. Regarding the cutoff distance $\left(r_{c}\right)$, only those bilayer atoms below the tip interact most strongly with the tip atoms while outside this region, the interaction strength decreases very fast. Therefore, in practice, the sum over $N_{b}$ can be truncated and limited to the atoms below the tip. This is done by employing a neighbor list in our molecular-dynamics simulation. The upper (bottom) layer atoms of the bilayer, below the tip region, interact with maximally $N_{t} \sim 300(250)$ tip atoms for small deflections and $N_{t} \sim 320(265)$ for large deflections. Moreover, the first term in Eq. (4), i.e., $k=1$ in the parentheses, is the derivative of the attractive part of the LJ potential and the second term $(k=2)$ is related to the derivative of the repulsive term. We will compare our obtained Young's modulus with those measured in experiments on graphene and graphite ${ }^{4,22}$ and compare our simulated force-deflection curves of the clamped circular bilayer graphene with those obtained for monolayer graphene. ${ }^{23}$ Circular red data in Fig. 2 show the variation in the applied load at $r=0$ as a function of the deflection in the $z$ direction. The fluctuation in the data are larger than those in our previous study on monolayer graphene. The reason is that in bilayer graphene both layers vibrate (due to thermal fluctuations) almost independently.

The plotted force was obtained as follows. After each 5000 time steps, the tip is pushed down with $0.2 \AA$ in order to induce the deflection $\zeta$. During this time interval, we let the system equilibrate and in the last 1000 time steps of these intervals, we calculate $F$ and obtain the mean value of $F$. Thus in a simulation with $10^{6}$ time steps, we have 200 points in Fig. 2. The tip atoms are closer to the upper graphene layer and the other layer is, on the scale of the tip interaction potential, far from the tip. The latter layer is more free to vibrate and it has a smaller interaction with the tip atoms. Therefore, the contribution of the bottom layer to the summation of Eq. (4) is less than the contribution of the other layer. In our simulation, we did not observe any defect formation even for large deflections. Moreover, all the bilayer data of Figs. 2 and 5 show steps in the force-deflection curves, that correspond to structural relaxation in the process of indentation. The reason is that the number of bilayer atoms which repel the tip are almost constant during the indentation steps. The reason that the bilayer is a discrete sheet and when it is indented by $2 \AA$ the number of tip neighbors (from bilayer atoms), which interact with the tip, are changed noncontinuously. Such steps can be smoothed by time averaging. As we see the curve for $R=12 \mathrm{~nm}$ is much smoother (than the two others in Fig. 2) since it was averaged over six simulations with different initial velocities. 
TABLE I. Values of $a$ and $b$ determined by fitting Eq. (3) to our simulated data given in Fig. 2. Values of $a^{\prime}$ and $b^{\prime}$ are determined by fitting separately the linear and cubic equations with the small and large deflection regions, respectively. The parameters $a_{m}$ and $b_{m}$ and also $a_{m}^{\prime}$ and $b_{m}^{\prime}$ for monolayer graphene which we determined earlier (Ref. 23) are also shown.

\begin{tabular}{ccccc}
\hline \hline $\begin{array}{c}R \\
(\mathrm{~nm})\end{array}$ & $\begin{array}{c}a \\
(\mathrm{~N} / \mathrm{m})\end{array}$ & $\begin{array}{c}b \\
\left(10^{17} \mathrm{~N} / \mathrm{m}^{3}\right)\end{array}$ & $\begin{array}{c}a^{\prime} \\
(\mathrm{N} / \mathrm{m})\end{array}$ & $\begin{array}{c}b^{\prime} \\
\left(10^{17} \mathrm{~N} / \mathrm{m}^{3}\right)\end{array}$ \\
\hline 12 & $7.10 \pm 0.4$ & $20.20 \pm 0.5$ & $7.15 \pm 0.9$ & $23.3 \pm 0.6$ \\
15 & $3.80 \pm 0.5$ & $15.60 \pm 0.2$ & $7.17 \pm 1.3$ & $16.77 \pm 0.8$ \\
18 & $4.60 \pm 0.5$ & $10.90 \pm 0.3$ & $3.19 \pm 0.9$ & $11.99 \pm 1.1$ \\
25 & $5.50 \pm 0.3$ & $4.20 \pm 0.2$ & $5.30 \pm 0.9$ & $6.04 \pm 0.9$ \\
& & & & $b_{m}^{\prime}$ \\
$R$ & $a_{m}$ & $b_{m}$ & $(\mathrm{~N} / \mathrm{m})$ & $\left(10^{17} \mathrm{~N} / \mathrm{m}^{3}\right)$ \\
$(\mathrm{nm})$ & $(\mathrm{N} / \mathrm{m})$ & $\left(10^{17} \mathrm{~N} / \mathrm{m}^{3}\right)$ & $13.48 \pm 0.1$ \\
12 & $5.21 \pm 0.08$ & $10.30 \pm 0.07$ & $3.41 \pm 0.1$ & $13.07 \pm 0.3$ \\
15 & $3.16 \pm 0.06$ & $7.07 \pm 0.01$ & $2.65 \pm 0.3$ & $10.07 \pm 0.1$ \\
18 & $3.06 \pm 0.04$ & $4.46 \pm 0.02$ & $1.86 \pm 0.1$ & $5.30 \pm 0.2$ \\
25 & $2.53 \pm 0.07$ & $2.70 \pm 0.05$ & $1.50 \pm 0.2$ & $4.01 \pm 0.2$ \\
30 & $1.30 \pm 0.09$ & $2.03 \pm 0.06$ & $0.65 \pm 0.1$ & $2.30 \pm 0.2$ \\
\hline \hline
\end{tabular}

The numerical results in Fig. 2 are fitted to the expression given by Eq. (3) and are shown by the solid curves on the circular red data. The values for the fitting parameters $a$ and $b$ are presented in Table I. The results in Fig. 2 show clearly the size dependence of the force-displacement curves and its difference from similar results for monolayer graphene which are shown by triangular green data in each panel of Fig. 2. The behavior of the tip-graphene flake interaction even for a few angstrom displacement can be understood from our simulation given here. To obtain quantitative results, we start with Eqs. (2) and (3) to describe the dependence of parameter $b$ on the radius for only large deflections. It is easy to obtain an analytical expression for the $b$ value for a large circular sample ${ }^{20}$ which is given by

$$
b \cong \frac{\pi E t}{4 G(\nu)} \frac{1}{R^{2}} .
$$

Note that the parameter, $a$, in Eq. (3) is equivalent to an effective spring constant for perpendicular indentation of the bilayer graphene when one assumes the bilayer graphene as an elastic membrane. Furthermore, as can be seen from Fig. 2 , for small deflection the forces are almost linear, we used a linear fit $\left[F_{l}(\zeta)=a^{\prime} \zeta\right]$ for small deflections $(\zeta \leq 1 \mathrm{~nm}$ on the order of $\sqrt{a / b})$ and for large deflections $(\zeta>1 \mathrm{~nm})$, we used a cubic fit $\left[F_{c}(\zeta)=b^{\prime} \zeta^{3}+C\right]$. The obtained values for the parameter $a^{\prime}$ and $b^{\prime}$ are listed in Table I. For $R=12,15$, and 18 $\mathrm{nm}$, the parameter $C$ is $11.3 \mathrm{nN}, 8.0 \mathrm{nN}$, and $6.5 \mathrm{nN}$, respectively, which are on the order of $a$.

The fitted curves are also shown in Fig. 2 by dasheddotted curves. Figure 3 shows the obtained $b$ and $b^{\prime}$ values as a function of the bilayer graphene circular size. The fitted parameter $b^{\prime}$ follows very well a $1 / R^{2}$ function and the agreement is now better than for the previous $b$ values.

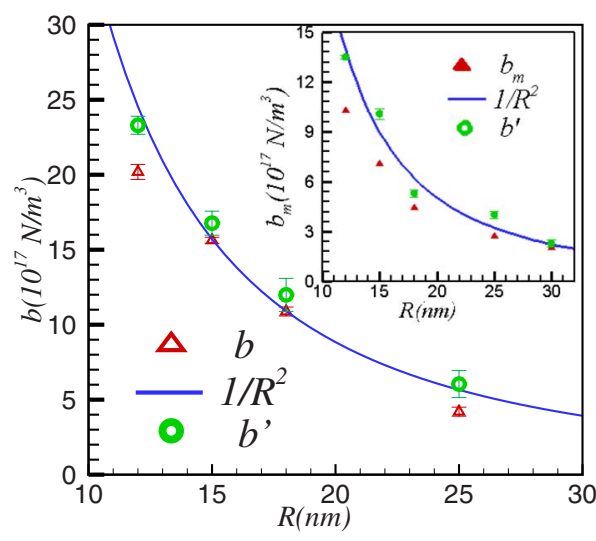

FIG. 3. (Color online) The parameters $b$ and $b^{\prime}$ as a function of the circular size of bilayer graphene. The inset shows the parameters $b_{m}$ and $b_{m}^{\prime}$ as a function of the circular size of monolayer graphene. In both cases, the solid curves show a $1 / R^{2}$ function fitted to the $b^{\prime}$ and $b_{m}^{\prime}$ data. For the definition of $b, b_{m}$ and $b^{\prime}, b_{m}^{\prime}$, we refer to the text. [i.e., Eqs. (3) and (5), respectively.]

For monolayer graphene, we fitted the function $F=a_{m} \zeta$ $+b_{m} \zeta^{3}$ to the results reported in our previous study. ${ }^{24}$ The corresponding values for $a_{m}$ and $b_{m}$ are listed in Table I. The fitted curves are shown by solid curves. Analogous as for bilayer graphene, we also used separate fits for small and large deflection: $F_{l}(\zeta)=a_{m}^{\prime} \zeta$ and $F_{c}(\zeta)=b_{m}^{\prime} \zeta^{3}+C^{\prime}$, respectively, which are presented in Table I. The corresponding fitted curves are shown by the dashed-dotted curves in Fig. 2 . For $R=12,15$, and $18 \mathrm{~nm}$, the parameter $C^{\prime}$ is $8.1 \mathrm{nN}$, $3.7 \mathrm{nN}$, and 3.9. $\mathrm{nN}$, respectively, which are on the order of $a^{\prime}$.

Furthermore, we fitted the values for $b^{\prime}$ to Eq. (5) and obtained $\pi E t / G(\nu) \approx 4 \times 353.5 \mathrm{~N} / \mathrm{m}$. This result is obtained from the function $G(\nu)=-0.59 \nu+0.94$ obtained from Ref. 20 and using the value $\nu \approx 0.25$ which is a typical Poisson's ratio for monolayer graphene. ${ }^{21}$ To obtain the Young modulus, we need an appropriate value for the thickness of bilayer graphene. One can estimate $t$ as the distance between the two layers plus the thickness of a monolayer. For monolayer graphene, some experimentalists used the value $0.23 \AA$ by assuming Young's modulus of graphene to be the same as in graphite. ${ }^{2}$ Others use different values for the thickness of monolayer graphene. Saitoh et al. estimated $0.874 \AA$ which was obtained from the parameters of the Brenner potential. ${ }^{24}$ This is an independent and more accurate value. Using this value, the bilayer thickness becomes $4.5 \AA$ which leads to the Young modulus $0.8 \mathrm{TPa}$ which is close to the values for graphite [i.e., 1.02 $\pm 0.03 \mathrm{TPa}$ (Ref. 22)] and those, found in recent experiments and found in theoretical calculations, for graphene [i.e., $1.0 \pm 0.1 \mathrm{TPa}$ (Ref. 4)].

In order to investigate the effects of having two instead of a single graphene layer, we calculated the ratio $\frac{F_{\text {bilayer }}}{F_{\text {monolayer }}}$ which we show in Fig. 4. For small indentation, the fluctuations in our results are larger because both layers vibrate more freely while in the large deflection regime, the elastic membrane energy is dominant. Furthermore, because of the nature of the LJ potential, the repulsion of bilayer graphene with the tip is more or less two times larger than for single monolayer 


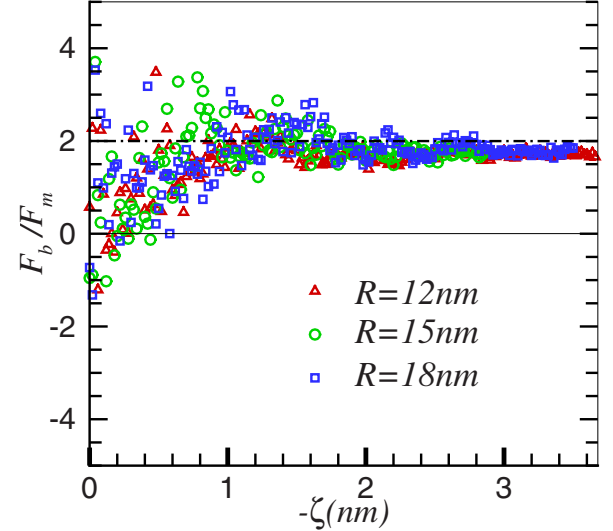

FIG. 4. (Color online) The ratio between the forces in loaded bilayer graphene and loaded monolayer graphene for three values of the size of the membrane.

graphene (see Fig. 4). As can be seen from Fig. 4, for small deflection where the Hooke's regime is dominant, we see a large difference between the spring constant ( $a$ parameters) of bilayer graphene and monolayer graphene. In the linear regime in Fig. 4, we have $\frac{F_{\text {bilayer }}}{F_{\text {monolayer }}} \approx \frac{a_{b}}{a_{m}}$, where $a_{b}(=a)$ and $a_{m}$ are the effective spring constant of bilayer and monolayer graphene. Figure 4 shows that in the small $\zeta$ region, we have on the average $F_{\text {bilayer }}<2 F_{\text {monolayer }}$ while in the large deflection regime, $F_{\text {bilayer }} \simeq 2 F_{\text {monolayer }}$. Note that the ratio $\frac{F_{\text {bilayer }}}{F_{\text {monolayer }}}$ in the large deflection limit is not exactly 2 because the bottom layer of bilayer graphene is far from the tip with respect to the upper layer so the number of interacting atoms in bilayer graphene to the tip at fixed deflection is not exactly twice those of monolayer graphene. Note that for small deflection (when the sheet is not stressed), shoulders (with small upwards amplitudes) are seen in both layers of the bilayer (particularly in the bottom layer because it is more free) around and below the tip which attract (they are close to the tip) the tip and cause negative forces even for small positive deflections (as measured from below the tip). This attraction dominates the repulsion term that is due to the other atoms below the tip. This unusual behavior is more clearly seen for larger systems.

At low temperature $(T=20 \mathrm{~K})$, graphene has a smaller roughness and behaves as a flat honeycomb lattice. One can compare the low-temperature data in Fig. 5 (an almost flat bilayer graphene with $R=12 \mathrm{~nm}$ ) to those for room temperature. At low temperature and for a fixed value of the displacement, the forces are smaller than those at room temperature. We fitted Eq. (3) to the data and found $a_{l}$ $=2.45 \mathrm{~N} / \mathrm{m}$ and $b_{l}=18.5 \times 10^{17} \mathrm{~N} / \mathrm{m}^{3}$. Using Eq. (5) yields

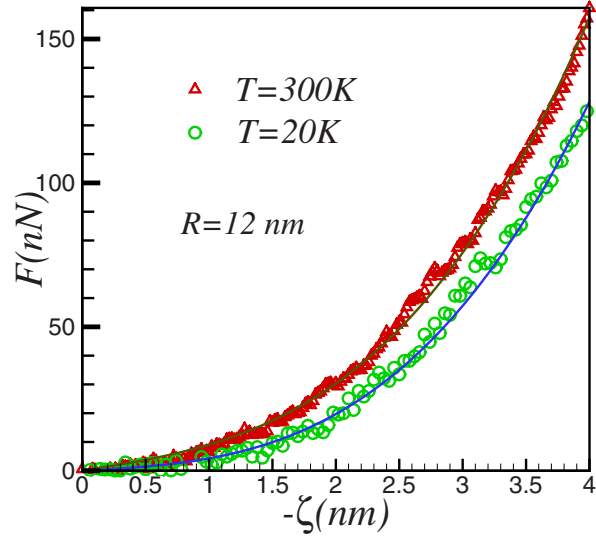

FIG. 5. (Color online) Comparison between the force-deflection response for rough (i.e., $T=300 \mathrm{~K}$ ) and almost flat (i.e., $T=20 \mathrm{~K}$ ) bilayer graphene for a membrane with radius $R=12 \mathrm{~nm}$. Solid curves are the fitted results using Eq. (3) with fitting parameters $a$ $=7.1 \mathrm{~N} / \mathrm{m}$ and $b=20.20 \times 10^{17} \mathrm{~N} / \mathrm{m}^{3}$ for $T=300 \mathrm{~K}$ and also $a_{l}$ $=2.45 \mathrm{~N} / \mathrm{m}$ and $b_{l}=18.5 \times 10^{17} \mathrm{~N} / \mathrm{m}^{3}$ for $T=20 \mathrm{~K}$.

Young's modulus $0.69 \mathrm{TPa}$ for low temperature which is $14 \%$ smaller than Young's modulus for bilayer graphene at room temperature. Such an increase in the Young's modulus with temperatures is unusual but it is similar to what has been reported recently by Zakharchenko et al. ${ }^{8}$ for monolayer graphene. This unusual behavior in both monolayer and bilayer graphene is the consequence of strong anharmonicity in graphene.

\section{CONCLUSION}

In this study, we showed that bilayer graphene like monolayer graphene and other carbon nanostructures has an exceptional stiffness. Young's modulus for bilayer graphene was calculated and found to be $0.8 \mathrm{TPa}$. The forcedisplacement result could be fitted to the function $F=a \zeta$ $+b \zeta^{3}$. We found that for given displacement, the exerted force on bilayer graphene has to be about twice the one on monolayer graphene. The force-deflection result is found to be temperature dependent. At low temperature, Young's modulus is found to be $14 \%$ smaller than at room temperature.

\section{ACKNOWLEDGMENTS}

We gratefully acknowledge comments from R. Asgari. M.N.-A. would like to thank the Universiteit of Antwerpen for its hospitality where part of this work was performed. This work was supported by the Flemish science foundation (FWO-Vl) and the Belgium Science Policy (IAP).
${ }^{1}$ A. K. Geim and K. S. Novoselov, Nature Mater. 6, 183 (2007);

A. H. Castro Neto, F. Guinea, N. M. Peres, K. S. Novoselov, and A. K. Geim, Rev. Mod. Phys. 81, 109 (2009).

${ }^{2}$ T. J. Booth, P. Blake, R. R. Nair, D. Jiang, E. W. Hill, U.
Bangert, A. Bleloch, M. I. Gass, K. S. Novoselov, M. I. Katsnelson, and A. K. Geim, Nano Lett. 8, 2442 (2008).

${ }^{3}$ K. S. Novoselov, A. K. Geim, S. V. Morozov, D. Jiang, Y. Zhang, S. V. Dubonos, I. V. Grigorieva, and A. A. Firsov, 
Science 306, 666 (2004).

${ }^{4}$ C. Lee, X. Wei, J. W. Kysar, and J. Hone, Science 321, 385 (2008).

${ }^{5}$ I. W. Frank, D. M. Tanenbaum, A. M. van der Zander, and P. L. McEuen, J. Vac. Sci. Technol. B 25, 2558 (2007).

${ }^{6}$ J. S. Bunch, A. M. van der Zande, S. S. Verbridge, I. W. Frank, D. M. Tanenbaum, J. M. Parpia, H. G. Craighead, and P. L. McEuen, Science 315, 490 (2007).

${ }^{7}$ J. S. Bunch, S. S. Verbridge, J. S. Alden, A. M. van der Zande, J. M. Parpia, H. G. Craighead, and P. L. McEuen, Nano Lett. 8, 2458 (2008).

${ }^{8}$ K. V. Zakharchenko, M. I. Katsnelson, and A. Fasolino, Phys. Rev. Lett. 102, 046808 (2009).

${ }^{9}$ S. N. Medyanik, E. G. Karpov, and W. K. Liu, J. Comput. Phys. 218, 836 (2006).

${ }^{10}$ A. Hemmasizadeh, M. Mahzoona, E. Hadia, and R. Khandana, Thin Solid Films 516, 7636 (2008).

${ }^{11}$ X. Xu and K. Liao, Mater. Phys. Mech. 4, 148 (2001).

${ }^{12}$ W. H. Duan and C. M. Wang, Nanotechnology 20, 075702 (2009).

${ }^{13} \mathrm{~S}$. Timoshenko and S. W. Krieger, Theory of Plates and Shells
(McGraw-Hill, New York, 1984).

${ }^{14}$ D. W. Brenner, Phys. Rev. B 42, 9458 (1990).

${ }^{15}$ N. Abedpour, M. Neek-Amal, R. Asgari, F. Shahbazi, N. Nafari, and M. R. Tabar, Phys. Rev. B 76, 195407 (2007); M. NeekAmal, R. Asgari, and M. R. Rahimitabar, Nanotechnology 20, 135602 (2009).

${ }^{16}$ Ş. Erkoç, Annual Reviews of Computational Physics IX, edited by D. Stauffer (World Scientific, 2001), pp. 1-103.

${ }^{17}$ H. A. Steel, The Introduction of Gases with Solid Surfaces (Pergamon, Oxford, 1974).

${ }^{18}$ R. Takagi, K. Kawamura, and M. Sakawa, J. Mater. Sci. Lett. 6, 217 (1987).

${ }^{19}$ L. D. Landau, L. P. Pitaevskii, A. M. Kosevich, and E. M. Lifshitz, Theory of Elasticity (Pergamon, Oxford, 1986).

${ }^{20}$ J. Cong-rui, Appl. Math. Mech. 29, 889 (2008).

${ }^{21}$ E. Cadelano, P. L. Palla, S. Giordano, and L. Colombo, Phys. Rev. Lett. 102, 235502 (2009).

${ }^{22}$ O. L. Blakslee, J. Appl. Phys. 41, 3373 (1970).

${ }^{23}$ M. Neek-Amal and R. Asgari, arXiv:0903.5035 (unpublished).

${ }^{24}$ K. Saitoh and H. Hayakawa, Phys. Rev. B 81, 115447 (2010). 